\title{
CHAITÉN: APRENDIZAJES DE UNA EXPERIENCIA DE DESASTRE SOCIONATURAL EN LA PATAGONIA CHILENA
}

\author{
EDITORIAL \\ ADRIANA ESPINOZA
}

Este número especial surge desde un compromiso de compartir los estudios sobre los efectos de la erupción del volcán Chaitén realizados por investigadores del Centro de Investigación en Vulnerabilidades y Desastres Socionaturales (CIVDES) ${ }^{1}$ de la Universidad de Chile y otros investigadores invitados. El objetivo es presentar algunos aportes a la comprensión de este tipo de eventos, enfatizando la importancia de estudiarlos desde las particularidades contextuales de cada desastre. En el caso de Chaitén, se hace imprescindible una comprensión desde su ubicación geográfica. Así como también, desde las políticas de gestión del riesgo que se han desplegado desde el inicio de la erupción en mayo de 2008, hasta el 2015 incluido. Esta información nos permite comprender, entre otras cosas, el impacto de estas medidas en las experiencias de evacuación, desplazamiento, retorno de su población y posterior reconstrucción material y psicosocial. A partir de este caso emblemático de evacuación, pretendemos también instalar una reflexión y debate respecto a la necesidad de adaptar las políticas de gestión del riesgo en desastres, incorporando la participación de las comunidades involucradas, para asegurar que las necesidades de los afectados estén siendo escuchadas y satisfechas.

En el primer artículo, Romero y Romero proporcionan un contexto geográfico y político desde el análisis de la erupción de los volcanes Hudson y Chaitén en la Patagonia. Situándose desde la ecología política, los autores reflexionan sobre cómo la falta

1 Núcleo Milenio de Investigación en Ciencias Sociales NS 100022, financiado entre los años 2012 y 2014 por la de información sobre estos territorios, ha incidido en la vulnerabilidad y la exclusión social y territorial de su población. Según estos autores, la actuación de instituciones públicas ha tenido consecuencias políticas y ecológicas negativas aumentando los niveles de vulnerabilidad de su población.

A partir de los antecedentes geológicos disponibles después de la erupción de año 2008, Lara y Calderón plantean que el volcán Chaitén es uno de los 10 volcanes más activos del territorio, colocando a la ciudad en una situación de riesgo dada su cercanía. Estos autores enfatizan la relevancia de una adecuada planificación del uso del territorio en los procesos de reconstrucción, considerando los antecedentes geológicos disponibles para así mitigar el impacto de futuros eventos eruptivos.

Desde la noción de justicia ambiental, Sandoval et al. analizan las decisiones gubernamentales diseñadas para el retorno de la población. Enfatizando que tanto la improvisación con que las autoridades enfrentaron la evacuación así como la indecisión del Estado sobre la reubicación de la localidad, han contribuido a que Chaitén se constituya en una ciudad desigual dado que los efectos de las políticas públicas se han distribuido de manera inequitativa en su reconstrucción, anticipando posibles situaciones de vulnerabilidad para su población.

El asentamiento permanente ha impactado también a las comunidades evacuadas. Berroeta et al. abordan los efectos del desplazamiento en las relaciones socioespaciales vinculadas al cambio de

Iniciativa Científica Milenio y el Ministerio de Economía, Fomento y Turismo del Gobierno de Chile. 
ambiente residencial en chaiteninos desplazadas que actualmente habitan en diversas localidades de las provincias de Palena y Llanquihue. Demostrando cambios significativos en los niveles de apego de lugar e identidad; sentido de comunidad; participación y satisfacción residencial en relación a su barrio de origen en Chaitén, y su lugar de residencia actual.

A partir de un diagnóstico participativo, Espinoza, Espinoza y Fuentes indagan acerca del proceso de desplazamiento y retorno de la comunidad educativa de la escuela Juan José Latorre ${ }^{2}$. Evidenciando la importancia de la escuela como eje central para el retorno de muchas familias; destacando también la importancia de estudiar los efectos de estos eventos en la salud mental de la comunidad educativa; y analizando la pertinencia de usar metodologías participativas en los procesos de reconstrucción psicosocial post-desastres socionaturales.

Mardones y Velásquez utilizan una metodología de Investigación-Acción-Participativa (IAP) para trabajar con jóvenes de la Escuela Juan José Latorre a través de talleres de radio. El objetivo era incorporar la comunicación comunitaria como una herramienta de reconstrucción social enfatizando el desarrollo de competencias, redes y visibilizando la participación de los jóvenes en este medio local. Estos talleres contribuyeron al fortalecimiento $y$ apoyo comunitario y a la creación de espacios de socialización. Reforzando también la pertinencia de estrategias metodológicas de participación comunitaria.

A nivel de estrategias de organización comunitaria, el trabajo de Berezin, presenta las razones que motivaron a algunos chaiteninos a articular un Movimiento Socioterritorial a través de prácticas de resistencia para la recuperación y reconstrucción de su pueblo, tras la erupción volcánica. A diferencia de otros movimientos socioterritoriales de la región, el autor plantea que los miembros de este Movimiento demostraron un fuerte arraigo y pertenencia a su tierra como parte de su identidad cultural.

Complementando el artículo anterior, Arteaga y Ugarte analizan los cambios y fracturas de los referentes identitarios de los chaiteninos producidos por las experiencias del desplazamiento y retorno. Es en este nuevo contexto territorial donde surgen

2 Agradecemos al entonces director don Raúl Jara y a todos los funcionarios de la Escuela Juan José Latorre por su in- otras formas de construir y negociar las identidades influyendo en la construcción de un nosotros, y en las transformaciones de sus expectativas y proyectos de vida.

En relación a las políticas públicas implementadas en Chaitén, Larenas, Salgado y Fuster plantean que estas políticas de Estado desaprovecharon los diversos capitales existentes, como redes basadas en la confianza y reciprocidad (capital social); sistemas productivos basados en los recursos territoriales (capital económico); y población que reconoce sus tradiciones e identidades locales (capital cultural y simbólico). Concluyendo que las agencias estatales actúan con esquemas estandarizados y centralistas apartados de las cosmovisiones existentes en territorios como la Patagonia, reflejando un desconocimiento de la pertinencia sociocultural y eficacia en sus actuaciones.

Finalmente, Tapia presenta una reflexión sobre el efecto sesgado y limitado de las políticas públicas relacionadas con la vivienda. Desde un exhaustivo análisis de las políticas públicas, ejecutadas por el Estado, entrevistas con los afectados y fuentes secundarias, Tapia concluye que estas estrategias no logran dar cuenta de las necesidades y expectativas de la comunidad respecto de la vivienda. Este proceso, aún inconcluso, tiene serias limitaciones conceptuales en su comprensión, diseño y gestión, produciendo serios daños en la calidad de vida de los habitantes, debido a que la vivienda, no fue considerada en su valor intrínseco, sino más bien solo en su condición de bien patrimonial.

Estos artículos nos acercan a la comprensión de un proceso complejo donde se visibilizan a lo menos dos focos de atención. El primero relacionado con la implementación inapropiada de políticas públicas estandarizadas y centralizadas para la gestión del desastre en la Patagonia. El segundo se centra en la inclusión de las comunidades afectadas en la re-formulación de políticas públicas incorporando diferentes estrategias participativas que aborden temas de género, sociales, culturales, identitarios y territoriales. Esperamos, a través de estos artículos, haber contribuido a encontrar aprendizajes y nuevas perspectivas respecto a temáticas, aún presentes, en la discusión nacional cada vez que un desastre socionatural afecta nuestro país.

terés en participar en este proceso de construcción de un conocimiento compartido a partir de sus experiencias. 